\title{
A Incorporação de Corredores de Conectividade no Manejo de Florestas Industriais Utilizando a Heurística da RazãoR
}

\author{
José Mauro Magalhães Ávila Paz Moreira ${ }^{1}$ \\ Luiz Carlos Estraviz Rodriguez ${ }^{2,3}$
}

\begin{abstract}
Resumo: Modelos de planejamento florestal com restrições espaciais resultam em problemas matemáticos combinatoriais de difícil resolução. O procedimento mais apropriado, neste caso, é desenvolver métodos heurísticos, em vez das técnicas tradicionais de programação linear. O objetivo deste trabalho é aplicar, aprimorar e avaliar o desempenho da heurística denominada RazaoR (NOBRE e RODRIGUEZ, 2005), desenvolvido para a solução de problemas de planejamento florestal formulados integralmente com variáveis binárias e de acordo com o modelo Tipo I (JOHNSON e SCHEURMAN, 1977 apud CLUTTER et al., 1992, p. 281). O método incorpora restrições espaciais de conectividade entre os fragmentos de vegetação nativa adjacentes às florestas de produção. $\mathrm{O}$ déficit de conectividade das soluções foi avaliado a partir de um algoritmo recursivo baseado na Teoria de Grafos e em técnicas de programação dinâmica. Como estudo de caso, utilizou-se uma fazenda de uma empresa de celulose do estado de São Paulo. A heurística foi eficaz e eficiente no número de iterações necessárias para encontrar medidas viáveis para o problema, mostrando-se ineficiente apenas no tempo computacional exigido. Os valores da função objetivo das soluções encontradas variaram de 99,71\% a 99,83\% do valor da solução ótima, com uma média de 48 iterações.
\end{abstract}

Palavras-chave: heurísticas, RazaoR, manejo florestal otimizado, corredores de conectividade, programação dinâmica.

Abstract: Planning models that connect natural forests through corridors formed by industrial forests result in combinatorial mathematical problems that are difficult to solve

1 Doutor em Economia Aplicada, Pesquisador da Embrapa Cerrados. E-mail: jose.moreira@cpac.embrapa.br, josemauropaz@gmail.com

2 Ph.D. Forest Management and Biometrics, Professor da Universidade de São Paulo, Escola Superior de Agricultura Luiz de Queiroz. E-mail: lcer@usp.br

3 Agradecimentos ao Conselho Nacional de Desenvolvimento Científico e Tecnológico (CNPq), e a Fundação de Amparo à Pesquisa do Estado de São Paulo (FAPESP), pelo apoio financeiro para a execução da pesquisa. 
and demand special techniques. The main objective of this paper is to apply, improve and evaluate the performance of a heuristic method named R-ratio (NOBRE and RODRIGUEZ, 2005), developed to solve forest planning problems that consider exclusively binary variables and are formulated as a model Type I harvest scheduling problem (JOHNSON and SCHEURMAN, 1977 apud CLUTTER et al., 1992, p. 281). The solution's connectivity deficit was evaluated by a recursive algorithm based on the Graph Theory and dynamic programming techniques. A farm of industrial forest that supplies round wood to a pulp wood company in the State of São Paulo was used as a study case. The method was effective in creating feasible solutions after a few number of interactions, showing inefficiency only in the computational time required. The objective function values of the solutions provided by the heuristic vary from $99.71 \%$ to $99.83 \%$ of the optimal solution found by a mixed integer linear programming, with an average of 48 interactions.

Key-words: heuristics, R-ratio, optimized forest management, connectivity corridors, dynamic programming.

Classificação JEL: Q2, Q23.

\section{Introdução}

As florestas sempre desempenharam papel fundamental na história da humanidade, dada a sua capacidade de manter a qualidade do ambiente e de, ao mesmo tempo, ofertar uma gama de produtos e serviços de vital importância para a sociedade (GARCIA e BORTOLETTO JÚNIOR, 2005). Tal reconhecimento cresceu a partir do final do século XX, sendo cada vez maior a cobrança para que o setor produtivo utilize técnicas de baixo impacto ambiental, aliando a produção de bens e serviços à conservação da biodiversidade.

Atender a essa requisição é o grande desafio, uma vez que a demanda por recursos naturais cresceu com o aumento significativo da população mundial (MEYER e TURNER, 1994), sendo necessário o uso de modernas técnicas para auxiliar os gestores florestais na sua tomada de decisão.

A consideração de questões ambientais e sociais nos critérios que norteiam o manejo florestal sustentável tem sido observada nas organizações brasileiras desde os anos 90, inclusive em empresas florestais do ramo de papel e celulose (AUGUSTI et al., 2005).

A incorporação de restrições ambientais, em especial aquelas envolvendo o manejo do espaço e da paisagem, tem se constituído em um dos campos de fronteira do conhecimento da pesquisa internacional na área de otimização do planejamento do manejo sustentado de florestas. O problema abordado mais frequentemente nesses estudos é a limitação da área máxima contínua de corte ${ }^{4}$

4 Conhecido como o problema de adjacência ou "Green-up Constraints". 
por período do horizonte de planejamento, que já se constituiu em norma de manejo sustentável para a Associação dos Produtores de Papel e Celulose dos países da América do Norte (AMERICAN FOREST AND PAPER ASSOCIATION, 2005). Outro ponto é a escolha de áreas para compor corredores de conectividade 5 entre fragmentos da paisagem. O problema de conectividade é conhecido por resultar em modelos de otimização da paisagem mais complexos (MARTELL, GUNN e WEINTRAUB, 1998).

O estabelecimento de corredores de conectividade entre fragmentos separados constitui-se em uma importante estratégia para permitir o aumento da população viável, facilitar o fluxo genético e conservar ambientes naturais. Isso porque possibilita a recolonização dos fragmentos com populações de tamanho reduzido, aumentando as chances de sobrevivência de várias espécies, além de reduzir a pressão do entorno sobre as áreas protegidas (ARRUDA, 2004; FONSECA et al., 2004).

A fragmentação do habitat consiste na remoção parcial de vegetação nativa para a instalação de agricultura, pecuária ou outras atividades humanas, isolando as populações da fauna e flora. Embora sejam mantidos fragmentos esparsos de vegetação nativa, dependendo da área e da espécie em questão pode não haver recursos naturais suficientes para garantir a sobrevivência no longo prazo de uma população com determinado número de indivíduos e variabilidade genética. Essa é a segunda principal causa do aumento da taxa de extinção das espécies observada a partir do século XX (PRIMACK e RODRIGUES, 2001).

A fragmentação reflete em perda de biodiversidade por três motivos principais. Além de limitar o potencial de uma espécie para dispersão e colonização, reduz a disponibilidade de alimento e outros recursos provindos das áreas naturais aos animais nativos, devido à diminuição de área. Essa descontinuidade pode, ainda, dividir populações em larga escala em duas ou mais subpopulações isoladas, as quais sofrerão uma perda de variabilidade genética, tornando-se mais susceptíveis à extinção.

Ao ligar fragmentos isolados por meio de corredores de conectividade, permite-se que indivíduos de uma mesma espécie, pertencentes a populações de fragmentos diferentes, possam interagir, construindo a chamada metapopulação.

Hess (1994) define metapopulação como "um conjunto de populações distribuídas por um número de fragmentos que são conectados, em graus variáveis, por movimentos de dispersão". A dinâmica da metapopulação depende da dinâmica das populações dentro dos fragmentos e da dinâmica das populações entre os fragmentos.

Embora nem sempre o corredor de conectividade resulte em um maior fluxo genético das populações que habitam os fragmentos conectados (HORSKHINS, MATHER e WILSON, 2006) - uma vez que esse depende também do compor-

5 Conhecido como o problema de conectividade. 
tamento das espécies -, existe certo consenso no meio científico que a sua implantação eleva a viabilidade das populações residentes (BEIER e NOOS, 1998). Assim, o estabelecimento de corredores emerge como uma condição necessária, mas não suficiente para o aumento da viabilidade das populações presentes em fragmentos de vegetação.

De modo geral, problemas de otimização do manejo sustentado de florestas que consideram questões espaciais apresentam natureza combinatorial, resultando em modelos de difícil resolução. Para isso, diversos métodos têm sido testados pela comunidade científica internacional.

Alguns autores sugerem algoritmos como a programação dinâmica (HOGANSON e BORGES, 1998; BORGES e HOGANSON, 1999), o algoritmo gerador de colunas $^{6}$ (WEINTRAUB, BARAHONA e EPSTEIN, 1994), ou, ainda, formulações de modelos de programação linear inteira mista (PLIM) (CERDEIRA et al., 2005; GOYCOOLEA et al., 2005; MCDILL e BRAZE, 2000, 2001; MCDILL, REBAIN e BRAZE, 2002; REBAIN e MCDILL, 2003; ÖNAL e BRIERS, 2005; WILLIAMS, 1998).

McDill e Braze (2001) afirmam que formulações de PLIM deveriam ser preferíveis, tendo em vista que são mais generalistas e podem ser solucionadas por softwares comerciais que utilizam os algoritmos branch and bound $(\mathrm{BBA})^{7} \mathrm{e}$ cutting plane. Tais programas estão em constante evolução, com versões mais elaboradas sendo disponibilizadas no mercado, permitindo a resolução de questões cada vez mais complexas.

Em problemas de manejo florestal de tamanho real, contudo, geralmente a dimensão das matrizes de dados e o número de variáveis inteiras ultrapassam a capacidade atual de resolução dos softwares comerciais em um tempo aceitável, o que leva outra parcela de autores a optar por métodos heurísticos. Mesmo sem uma solução ótima, esses métodos garantem uma redução expressiva do tempo computacional necessário para a resolução de modelos com restrições espaciais em escala real.

De acordo com Hillier e Lieberman (2005), a heurística é um procedimento desenvolvido para descobrir uma solução viável, mas não necessariamente ótima, do problema considerado. Geralmente, constitui-se de um algoritmo iterativo, no qual, a cada iteração, é realizada a busca por uma nova solução que seja melhor que a anteriormente sugerida. O algoritmo é encerrado após um determinado número de iterações, e a melhor solução obtida é apresentada.

As abordagens mais utilizadas para solucionar problemas de planejamento do manejo de florestas com restrições espaciais envolvem heurísticas desenvolvidas pelos próprios autores (CERDEIRA et al., 2005; FALCÃO e BORGES, 2002; LOEHLE, 1999; MURRAY, 1999; SESSIONS, 1992), heurísticas de uso

6 "Column Generation".

7 Algoritmo para solução de problemas de programação linear com variáveis inteiras. 
comum em outras áreas, como a Busca Tabu' (CARO et al., 2003), "simulated annealing" (LOCKWOOD e MOORE, 1993; ÖHMAN, 2000; ÖHMAN e ERIKSSON, 1998), e, ainda, um misto de programação linear e "simulated annealing" (ÖHMAN e ERIKSSON, 2002).

A resolução de problemas envolvendo corredores entre fragmentos florestais, por meio de métodos heurísticos, foi abordada nos trabalhos de Sessions (1992), Loehle (1999), Cerdeira et al. (2005) e Fuller e Sarkar (2006).

Sessions (1992) modelou a conectividade como um problema de rede, com as áreas chaves de habitat que deveriam ser preservadas sendo previamente designadas como um corredor e as unidades necessárias para conectar essas áreas sendo escolhidas a partir de uma rede Steiner ${ }^{9}$. Minimiza-se, portanto, a perda de madeira não colhida nas áreas designadas para compor o corredor.

Loehle (1999) propôs um algoritmo baseado na dispersão e no reconhecimento de odores, para encontrar o caminho mais curto ao conectar fragmentos isolados na paisagem. $\mathrm{O}$ algoritmo mostrou-se eficiente e eficaz não somente para ligar esses fragmentos como também para alterar o formato e aumentar a sua área interior.

Cerdeira et al. (2005) a plicaram uma heurística em três estágios para encontrar uma rede conectada na paisagem, contendo todas as espécies e minimizando o número de fragmentos. Os autores inseriram a conectividade como parte das restrições do problema e consideraram apenas soluções viáveis no seu algoritmo. A heurística apresentou-se eficaz e eficiente, apontando soluções viáveis muito próximas da solução ótima e com um esforço computacional inferior a um minuto, enquanto a solução ótima obtida pelo BBA levou mais de 11 horas para ser obtida.

Fuller e Sarkar (2006) desenvolveram um software para análise e implantação de corredores de conectividade entre áreas de conservação, visando à maior qualidade ambiental ou menor área. A conectividade entre os fragmentos foi representada como um grafo, e os autores utilizaram um algoritmo para encontrar as menores árvores que abrangessem todos os nós (fragmentos). $\mathrm{O}$ algoritmo mostrou-se capaz de lidar com problemas de conexão de áreas a serem conservadas bem maiores que os métodos tradicionalmente usados.

Os trabalhos citados anteriormente buscaram minimizar o custo da incorporação de conectividade ou maximizar a qualidade ambiental do corredor a ser estabelecido. No entanto, a literatura ainda encontra-se carente de estudos que mantenham os objetivos tradicionais do planejamento de manejo florestal, como a maximização dos lucros ou da produção, incorporando a formação de corredores de conectividade.

8 "Tabu Search".

9 O problema de rede Steiner difere do problema "the minimum spanning tree", pois não é necessário que todos os nós da rede estejam conectados. 
Rodriguez (1994) desenvolveu um novo processo heurístico para lidar com os problemas de manejo florestal com variáveis inteiras em larga escala, representado pelo Modelo Tipo I (JOHNSON e SCHEURMAN, 1977 apud CLUTTER et al., 1992, p. 281) e denominado heurística da RazaoR (NOBRE e RODRIGUEZ, 2005). Essa heurística foi aprimorada no trabalho de Nobre (1999), tanto no aspecto de sua lógica como na sua implantação computacional, mostrando-se um método promissor para resolução de problemas de manejo de florestas com variáveis inteiras.

A lógica que guia o processo de busca de novas soluções na heurística da RazaoR baseia-se na escolha da alternativa que apresente o menor custo unitário para a obtenção das metas de produção - ou seja, a opção com a menor queda no valor da função objetivo por unidade reduzida no déficit de produção.

A heurística da RazaoR se inicia com a solução de maior valor da função objetivo que pode ser obtido para o problema, escolhendo o regime de manejo de maior valor da função objetivo para cada unidade de manejo, sem se preocupar com o atendimento das metas (restrições) de produção. Em seguida, as variáveis de decisão (representadas pelos regimes de manejo) são separadas em três grupos distintos: as selecionadas (que constituem a base), as nãoselecionadas e as descartadas. O próximo passo é a análise da vizinhança da solução atual, realizada pela substituição, para cada unidade de manejo, de um regime de manejo pertencente ao grupo das variáveis não-selecionadas, pelo regime de manejo associado à unidade de manejo selecionada que se encontra na base da solução. Avalia-se, então, a redução no Valor Total do Projeto (VTP) e no déficit em relação às metas impostas pelas restrições (metas de produção anual). Posteriormente, estabelece-se uma razão entre a redução do déficit e a diminuição do VTP, sendo esta a RazaoR que guiará a busca. O processo é repetido para cada regime de manejo não-selecionado, para cada unidade de manejo do problema, de modo a criar a vizinhança da solução contida na base. Então, a heurística seleciona o regime de manejo associado a uma unidade de manejo que apresenta a maior RazaoR para entrar na base, inserindo-a no grupo das selecionadas, em substituição ao regime de manejo anteriormente escolhido, o qual será destinado para o grupo das variáveis descartadas.

Para melhorar e diversificar o processo de busca, a heurística visita o grupo das soluções descartadas periodicamente, com base em dois parâmetros: alfa (iteração na qual o grupo das descartadas é visitado pela primeira vez) e beta (número de iterações em que a visita se repete). A heurística procede dessa forma até encontrar uma solução com déficit zero, ou ultrapassar o número máximo de iterações definido pelo usuário, ou, ainda, sem ter como continuar o processo de busca.

Caso a heurística encontre uma solução com déficit zero, inicia-se, então, um novo processo chamado Fase Verde, no qual todas as variáveis fora da base são avaliadas a cada iteração, sendo consideradas aquelas que mantêm o déficit zero e aumentam o VTP, e escolhida a que proporciona o maior aumento do VTP. A 
Fase Verde termina quando não é encontrada nenhuma variável que, ao entrar na base, mantenha o déficit igual a zero e aumente o VTP, sendo esta a solução final apresentada pela heurística. Uma descrição detalhada do funcionamento da heurística da RazaoR, bem como uma avaliação do seu processo de busca à luz dos conceitos da inteligência artificial, está disponível nos estudos de Rodriguez (1994), Nobre (1999) e Nobre e Rodriguez (2005).

O desafio proposto neste trabalho consiste em sugerir os ajustes necessários para que a heurística da RazaoR possa lidar com o planejamento do manejo de florestas equiâneas considerando as metas anuais de produção em conjunto com a necessidade de se garantir corredores de conectividade entre fragmentos de vegetação nativa adjacentes a essas florestas. Para isso, será necessário o desenvolvimento de um método para mensurar o déficit de conectividade das soluções, bem como a sua inserção no algoritmo da heurística.

\section{Material e Métodos}

\subsection{Fonte de dados}

O estudo de caso deste trabalho considera uma fazenda pertencente a uma empresa de celulose e papel do estado de São Paulo, que será chamada de Fazenda Empresarial. A fazenda possui 6.262 hectares, sendo 1.571 ha ocupados com vegetação nativa, 4.419 ha com plantios de eucalipto e 272 ha com áreas de outros usos. A distribuição da área por classes de idade e por rotação atual dos plantios de eucalipto pode ser observada na Figura 1a; a Figura 1b apresenta a curva média de produção esperada, de madeira de celulose, dos talhões da fazenda em primeira e segunda rotações.

O mapa da Fazenda Empresarial, com a localização das unidades de manejo, dos fragmentos de floresta nativa e das áreas de outros usos, bem como os caminhos utilizados para a formação dos possíveis corredores, pode ser observado na Figura 2.

Figura 1. Distribuição de área por idade e rotação atual dos talhões (a) e a curva de prognose de produção (b) para a Fazenda Empresarial.
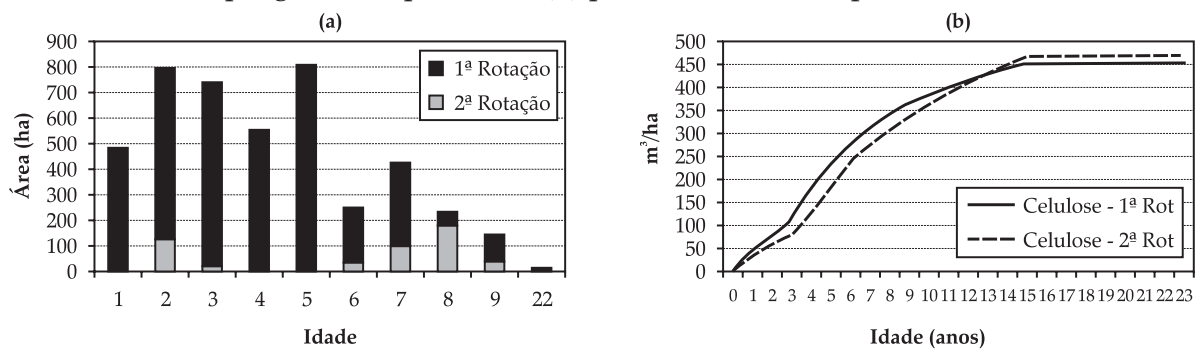

Fonte: Dados obtidos junto à empresa parceira no estudo. 
O planejamento da produção foi feito considerando um produto (madeira para celulose), com custo de oportunidade do capital de $10 \%$ ao ano. O preço da madeira de eucalipto em pé foi extraído do Informativo Cepea - Setor Florestal (2006), de R \$39,00/st em pé, valor que, dividido pelo fator de empilhamento de $0,7 \mathrm{~m}^{3} / \mathrm{st}$, resulta em $\mathrm{R} \$ 55,71 / \mathrm{m}^{3}$. As idades mínima e máxima para o corte da floresta foram de seis e nove anos, respectivamente. O horizonte de planejamento foi de 18 anos, com produção mínima anual de madeira para celulose, de $180.000 \mathrm{~m}^{3}$ e a máxima, de $250.000 \mathrm{~m}^{3}$.

Os regimes de manejo foram elaborados com duas rotações por ciclo de produção, havendo alternância entre ciclos dentro de um mesmo regime. $\mathrm{O}$ resultado foram 143 regimes de manejo para cada unidade de manejo, em média, totalizando 13.012 variáveis binárias a serem consideradas na heurística, sendo quatro dessas para a incorporação dos fragmentos.

Escolheu-se o Fragmento 1 como unidade de origem para a formação do corredor, devido à sua localização mais centralizada. Os demais fragmentos foram designados como unidades de destino, e as unidades de manejo foram definidas como unidades de passagem.

Em acordo com os técnicos da empresa, decidiu-se por considerar a idade do plantio como a característica que permitirá a escolha das unidades de manejo para compor o corredor em um determinado período - foi estipulada idade maior ou igual a três anos para tornar a unidade de manejo apta a fazer parte do corredor.

Figura 2. Mapa da Fazenda Empresarial.

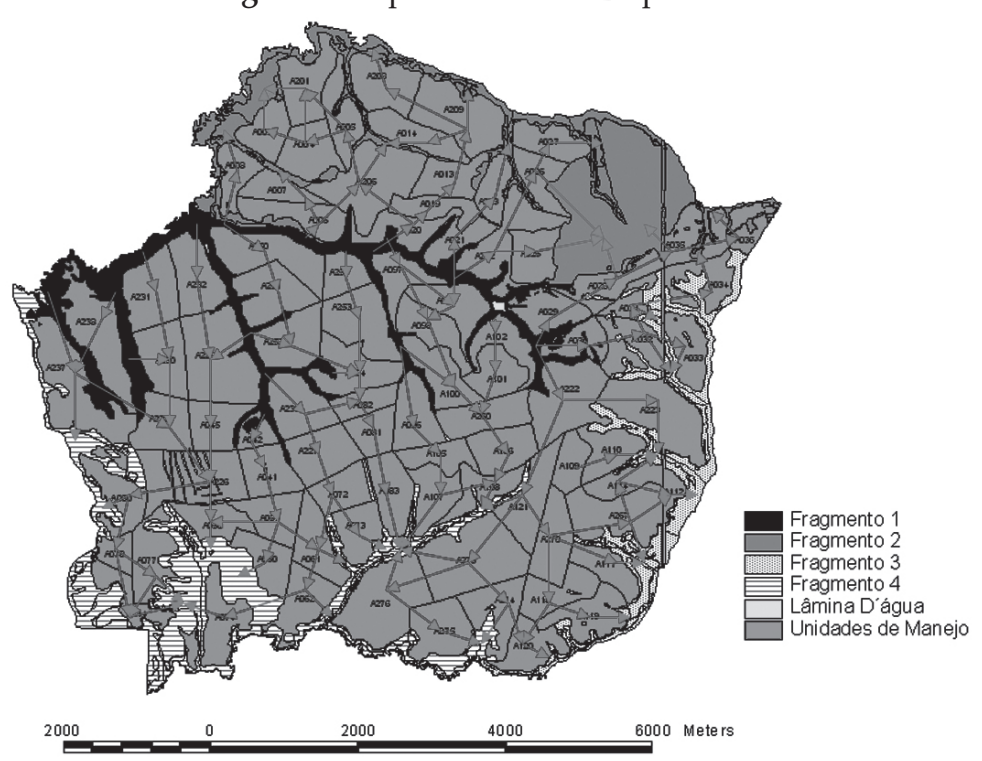

Fonte: Mapa obtido junto à empresa parceira no estudo. 


\subsection{Método proposto para avaliação do déficit de conectividade}

O método proposto utiliza um algoritmo recursivo apresentado no texto de Maurer e Ralston (2004), muito aplicado na teoria dos grafos para resolução de problemas de programação dinâmica (WINSTON, 2004) e de programação em redes (HILLIER e LIEBERMAN, 2005). A fórmula para calcular o déficit de conectividade de cada unidade de manejo é apresentada na equação (1).

$$
\operatorname{Def}_{\mathrm{It}}=\mathrm{b}_{\mathrm{IJt}} \hat{A R E A}_{\mathrm{I}}+\operatorname{Min}_{\mathrm{K}}\left\{\operatorname{Def}_{\mathrm{Kt}} \mid \mathrm{K} \in \mathrm{F}_{\mathrm{I}}\right\}
$$

Em que:

$\mathrm{I}, \mathrm{K}$ referem-se às unidades de manejo (UM); $\mathrm{J}$ aos regime de manejo $(\mathrm{RM}) ; \mathrm{t}$ aos períodos do horizonte de planejamento; $\mathrm{F}_{\mathrm{I}}$ representa o conjunto das UM's de manejo que possuem arcos entrando na $\mathrm{UM}_{\mathrm{I}}$; $\operatorname{Def}_{\mathrm{It}}$ acumula o déficit de conectividade, em hectares, do talhão I no período $t$; $b_{\mathrm{IJt}}$ refere-se ao parâmetro refletindo a condição da $\mathrm{UM}_{\mathrm{I}}$, submetido ao $\mathrm{RM}_{\mathrm{J}}$, no período $t$.

Ao proceder com o cálculo do déficit de conectividade (em hectares) de cada $\mathrm{UM}$, a variável $\operatorname{Def}_{\mathrm{It}}$ adiciona ao déficit da $\mathrm{UM}_{\mathrm{I}}$ o menor déficit acumulado nas UM's que apresentam arcos terminados na $\mathrm{UM}_{\mathrm{I}}$. Desse modo, quando calculado, o déficit de conectividade dos fragmentos de destino ( 2 a 4 ) representará o déficit de conectividade do caminho composto, entre o Fragmento de origem e o Fragmento de destino em questão, pelas UM's que apresentam a menor área possível com UM’s que não estariam aptas a fazer parte do corredor.

$O$ parâmetro $b_{\mathrm{IJt}}$ assume valores zero para UM's que possam fazer parte do corredor no período $t$, um para UM's que não possam fazer parte do corredor e não estejam sendo colhidas no período $t$, e dez para UM's que estejam sendo colhidas no período t. O objetivo de penalizar as UM's em colheita é não considerá-las no cálculo do déficit de conectividade, uma vez que tais atividades desestimulam fortemente o trânsito da fauna nessa UM durante a sua realização.

\subsection{Modelo utilizado para o cálculo da heurística}

As equações a seguir são necessárias para o processo de cálculo da heurística.

$$
\begin{array}{ll}
\operatorname{VTP}=\sum_{\mathrm{i}=1}^{\mathrm{n}} \mathrm{A}_{\mathrm{i}} \mathrm{D}_{\mathrm{ij}} \mathrm{X}_{\mathrm{ij}} & \mathrm{j} \in \mathrm{S} \\
\text { VTPaval }=\sum_{\mathrm{i}=1}^{\mathrm{n}} \mathrm{A}_{\mathrm{i}} \mathrm{D}_{\mathrm{ik}} \mathrm{X}_{\mathrm{ik}} & \mathrm{k} \in \mathrm{G} \\
\text { Prod }_{\mathrm{t}}=\sum_{\mathrm{i}=1}^{\mathrm{n}} \mathrm{A}_{\mathrm{i}} \mathrm{V}_{\mathrm{ij \textrm {t }}} \mathrm{X}_{\mathrm{ij}} & \mathrm{j} \in \mathrm{S}
\end{array}
$$


264 - A Incorporação de Corredores de Conectividade no Manejo de Florestas Industriais Utilizando a Heurística da RazãoR

$$
\begin{aligned}
& \operatorname{ProdAval}_{\mathrm{t}}=\sum_{\mathrm{i}=1}^{\mathrm{n}} \mathrm{A}_{\mathrm{i}} \boldsymbol{v}_{\mathrm{ikt}} \mathrm{X}_{\mathrm{ik}} \quad \mathrm{k} \in \mathrm{G} \\
& \operatorname{DefP}_{t}=\left\{\begin{array}{clc}
\text { Prod }_{t}-\text { Teto }_{t} & \text { se } & \text { Prod }_{t}>\text { Teto }_{t} \\
\text { Piso }_{t}-\text { Prod }_{t} & \text { se } & \text { Prod }_{t}-\text { Piso }_{t} \\
0 & \text { se Piso } & \leq \text { Prod }_{t} \leq \text { Teto }_{t}
\end{array}\right. \\
& \text { DefPAval }_{\mathrm{t}}=\left\{\begin{array}{clc}
\text { ProdAval }_{\mathrm{t}}-\text { Teto }_{\mathrm{t}} & \text { se } & \text { ProdAval }_{\mathrm{t}}>\text { Teto }_{\mathrm{t}} \\
\text { Piso }_{\mathrm{t}}-\text { ProdAval }_{\mathrm{t}} & \text { se } & \text { ProdAval }_{\mathrm{t}}<\text { Piso }_{\mathrm{t}} \\
0 & \text { se } \text { Piso }_{\mathrm{t}} \leq \text { ProdAval }_{\mathrm{t}} \leq \text { Teto }_{\mathrm{t}}
\end{array}\right.
\end{aligned}
$$

DefProd $=\sum_{\mathrm{t}=1}^{\mathrm{T}} \operatorname{DefP}_{\mathrm{t}}$

DefProdAval $=\sum_{\mathrm{t}=1}^{\mathrm{T}}$ DefPAval $_{\mathrm{t}}$

DefConect $=\sum_{\mathrm{I} \in \mathrm{E}} \sum_{\mathrm{t}=1}^{\mathrm{T}} \operatorname{DefC}_{\mathrm{Et}}$

DefConectAval $=\sum_{\mathrm{I} \in \mathrm{E}} \sum_{t=1}^{\mathrm{T}} \operatorname{DefCAval}_{\mathrm{Et}}$

$$
\Delta \mathrm{VTP}=\mathrm{VTP}-\mathrm{VTPAval}
$$

$\Delta$ Deficit $=$ Def Prod - Def ProdAval

+ PesoConec (DefConec - DefConecAval)

Pond $=\operatorname{DefTotal}_{\mathrm{Ini}} / \mathrm{VTP}_{\mathrm{Ini}}$

$$
\text { RazaoR }=\left\{\begin{array}{cl}
\Delta \text { Deficit } & \text { se }\{\Delta \text { Deficit } \geq 0, \Delta \text { VTP } \leq 0\} \\
\Delta \text { Deficit } / \Delta \text { VTP } & \text { se }\{\Delta \text { Deficit } \geq 0, \Delta \text { VTP }>0\} \\
\left(\text { Pond } / N^{\circ} \text { It }\right)(\Delta \text { VTP } / \Delta \text { Deficit }) & \text { se }\{\Delta \text { Deficit }<0, \Delta \text { VTP }<0\} \\
\text { SemMelhora } & \text { se }\{\Delta \text { Deficit }<0, \Delta \text { VTP } \geq 0\}
\end{array}\right.
$$

Em que $i$ representa o conjunto das Unidades de Manejo (UM); $j, k$, os regimes de manejo (RM); $t$ os períodos do horizonte de planejamento; $S$ o conjunto dos RM da solução selecionada a cada iteração; $G$ o conjunto dos RM pertencentes às variáveis do grupo avaliado na iteração, podendo ser das não-selecionadas ou das descartadas; $E$ o conjunto dos Fragmentos de destino dos corredores de conectividade; $A_{i}$ a área da unidade de manejo $i ; D_{i j}$ a contribuição para a função objetivo caso a unidade de manejo $i$ seja associada ao Regime de Manejo $j ; x_{i j}$ a variável binária que representa a escolha do $\mathrm{RM} j$ para a UM $i$; $v_{i j t}$ representa a 
produção de madeira da unidade de manejo $i$ no período $t$ quando associada ao Regime de Manejo j; VTP o valor total do projeto da solução selecionada; VTPAval o valor total do projeto de cada solução avaliada; $\operatorname{Prod}_{t}$ o volume produzido pela solução selecionada no período $t$; ProdAval $_{t}$ o volume produzido pela solução avaliada no período $t$; DefP $t_{t}$ o déficit de produção no período $t$ resultante da solução selecionada; Teto $_{t}$ representa a quantidade máxima de madeira a ser produzida no período $t$; Piso $_{t}$ a quantidade mínima de madeira a ser produzida no período $t$; DefPAval o déficit de produção no período $t$ resultante de cada solução avaliada; DefProd o déficit total de produção da solução selecionada; DefProdAval o déficit total de produção de cada solução avaliada; $D e f C_{E t}$ o déficit de conectividade acumulado em cada fragmento de destino, no período $t$, pela solução selecionada; DefCAval ${ }_{E t}$ o déficit de conectividade acumulado em cada fragmento de destino, no período $t$, por cada solução avaliada; DefConec o déficit total de conectividade (em hectares) da solução selecionada; DefConecAval o déficit total de conectividade (em hectares) de cada solução avaliada; $\triangle V T P$ a variação no valor total do projeto caso a solução avaliada seja escolhida como a próxima solução selecionada; PesoConec o parâmetro que serve para ponderar o valor do déficit de conectividade em relação ao déficit de produção; $\Delta$ Deficit a variação no valor total do déficit em relação às metas impostas pelas restrições, caso a solução avaliada seja escolhida como a próxima solução selecionada; SemMelhora é o parâmetro inserido pelo usuário para que as soluções avaliadas que correspondam à quarta condição do cálculo da RazaoR assumam - esse é um valor de referência que serve como regra de parada da heurística, assim como o número máximo de iterações; Pond representa o parâmetro de ponderação; DefTotal $_{I n i}$ o déficit total calculado para a solução inicial do problema; $V T P_{I n i}$ o Valor Total do Projeto para a solução inicial do problema; RazaoR a variável que guia o processo de escolha da heurística na busca de boas soluções viáveis; e №It o número de iterações efetuadas durante a busca.

As equações (2) e (3) calculam o valor total do projeto da atual seleção escolhida e da seleção candidata a cada iteração, respectivamente. As equações de (4) a (11) exercem o papel de avaliar o grau de não viabilidade das soluções selecionadas e avaliadas em relação às metas impostas pelas restrições. Vale salientar que, enquanto as equações (8) e (10) não atinjam valor zero, a heurística procede em sua busca em todo o espaço de soluções, tanto as viáveis como as inviáveis. Quando uma solução que zera essas duas equações é selecionada, a heurística inicia o processo de busca denominado Fase Verde, o qual restringe o espaço de soluções avaliadas àquelas que sejam viáveis. A equação (15) é a função de avaliação que guia o processo de busca da heurística, a qual procede com a busca, escolhendo sempre a variável avaliada com o maior valor da RazaoR para entrar na base e passar a compor o grupo das selecionadas.

Nobre (1999) ressalta que a RazaoR realiza sempre igual caminho de busca para um mesmo conjunto de parâmetros alfa e beta, retornando sempre à mesma 
solução. Uma maneira sistemática de fazer a heurística investigar diferentes caminhos, chegando a soluções distintas, é alterar, de forma organizada, o valor dos parâmetros alfa e beta.

Nessa nova formulação, torna-se necessária a inserção de um parâmetro para ponderar as diferentes restrições impostas ao problema, as metas de produção e a conectividade. Este seria um terceiro parâmetro a influenciar a rota de busca da heurística. Além disso, a equação (15) foi modificada em relação à função original apresentada por Rodriguez (1994) e Nobre (1999), especificamente na forma de avaliação para alternativas que registrem aumento no déficit de produção e no VTP. A lógica de guia para a escolha da melhor RazaoR baseia-se na seleção da alternativa que, proporcionalmente, reduza ao máximo o valor do déficit com o menor custo, avaliado em termos de redução da função objetivo, neste caso o VTP.

A terceira condição de cálculo da RazaoR, apresentada pela equação (15), possibilita que a heurística selecione soluções que aumentem o déficit em relação às metas, desde que essa solução aumente o VTP. Isso possibilita uma maior diversificação do processo de busca e uma recuperação do VTP ao longo do processo antes de se entrar na Fase Verde.

De modo a manter a lógica da heurística, o cálculo da terceira condição foi modificado, invertendo-se os termos da razão. Esse procedimento apontou um problema de escala entre as duas grandezas, sendo necessário inserir um ponderador para trazer o valor da RazaoR sob esta condição a patamares semelhantes às outras condições. O ponderador também foi divido pelo número de iterações do processo de busca, visando tornar as soluções que aumentam o déficit total menos atrativas, elevando a convergência da heurística. Essa estratégia foi implantada porque, segundo Nobre (1999), a heurística da RazaoR piora o valor da solução final à medida que o processo de busca se prolonga. A equação (15) apresenta a nova função de avaliação da RazaoR.

\subsection{O funcionamento da heurística}

O processo geral de busca da heurística pode ser observado na Figura 3. Inicialmente, a heurística obtém, a partir de planilha Excel, os dados referentes aos regimes de manejo para cada unidade de manejo (coeficiente da função objetivo, prognose de produção, parâmetros para o cálculo do déficit de conectividade), as unidades de manejo de origem e destino dos arcos direcionados pré-determinados para o estabelecimento dos corredores de conectividade e as metas anuais de produção e de conectividade estabelecidas. 


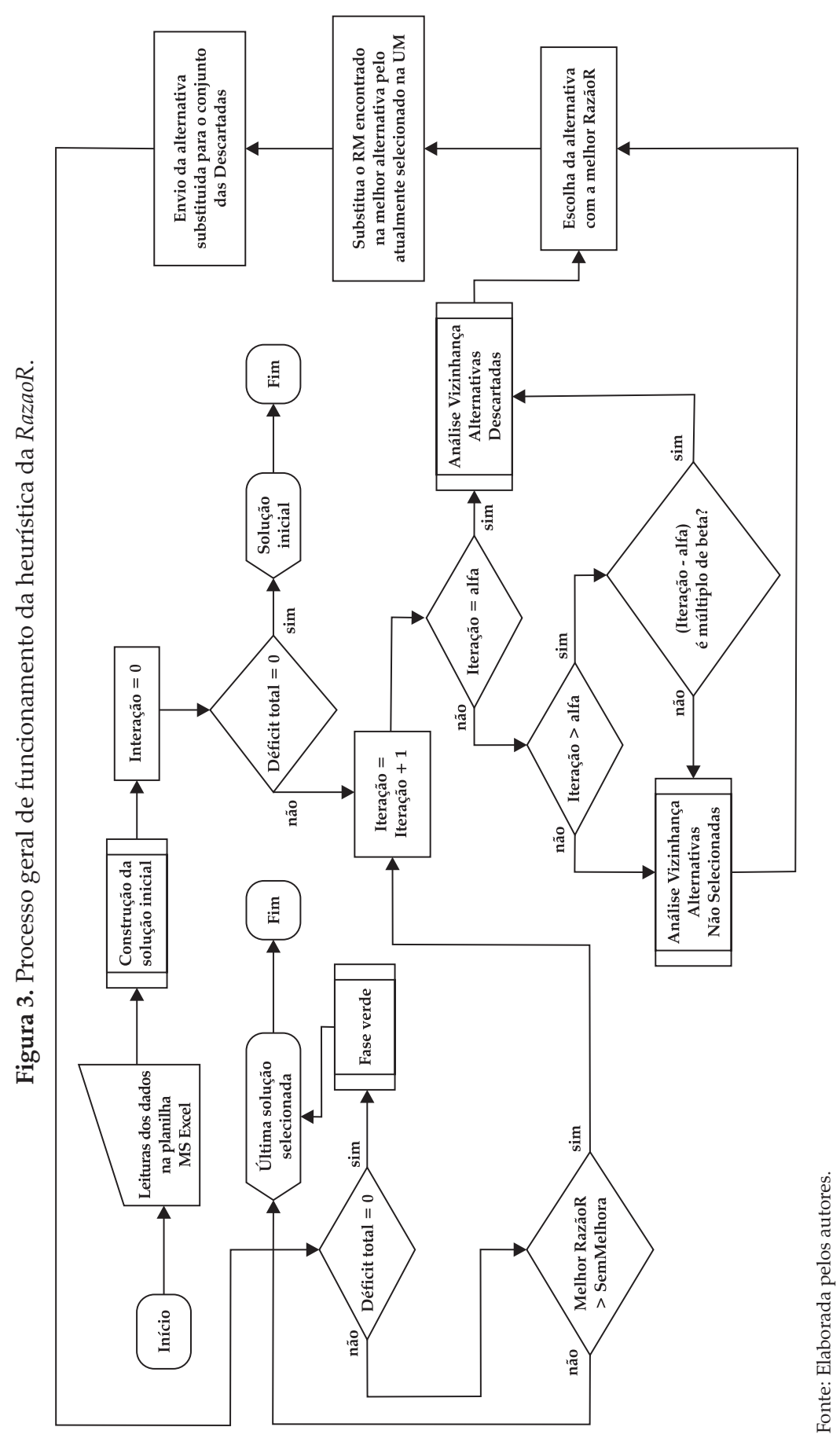

RESR, Piracicaba, SP, vol. 48, no 02, p. 255-282, abr/jun 2010 - Impressa em mês 2010 
Em seguida, elabora a solução inicial (Figura 4), escolhendo o regime de manejo cujo coeficiente da função objetivo seja o maior para cada unidade de manejo - sem se preocupar com o atendimento das restrições - e avaliando se a solução inicial atende às metas de produção e conectividade estabelecidas. Em caso afirmativo, tem-se a melhor solução para o problema, a qual é apresentada pela heurística, encerrando-se o processo de busca. Do contrário, inicia-se o processo de construção e análise da vizinhança da solução atual e a busca de soluções melhores que a atualmente armazenada na base do problema.

Figura 4. Processo de construção da solução inicial da heurística da RazaoR.

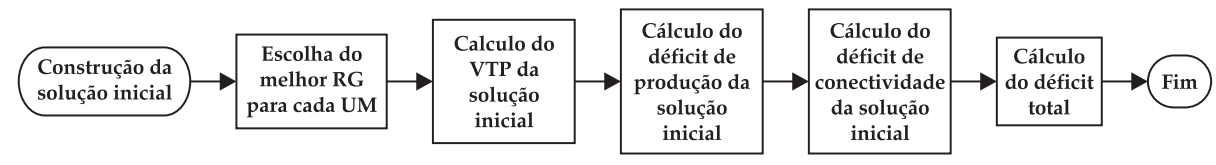

Fonte: Elaborada pelos autores.

O processo de busca por soluções melhores começa com o controle do número de iterações. Posteriormente, é feita a comparação com os parâmetros alfa e beta, de modo a avaliar se a vizinhança daquela iteração deve ser construída utilizando as variáveis presentes no conjunto das não-selecionadas ou das descartadas.

A primeira construção da vizinhança com as variáveis do conjunto das descartadas é realizada quando o número da iteração atual é igual a alfa, sendo esse conjunto utilizado novamente para a construção da vizinhança a cada beta iterações a partir de então. Em todas as outras iterações, a vizinhança da solução atual é construída a partir das variáveis do conjunto das alternativas não-selecionadas. O processo detalhado de construção da vizinhança por meio de alternativas não-selecionadas pode ser observado na Figura 5a. A Figura $5 b$ ilustra o processo de construção da vizinhança com as alternativas descartadas. 


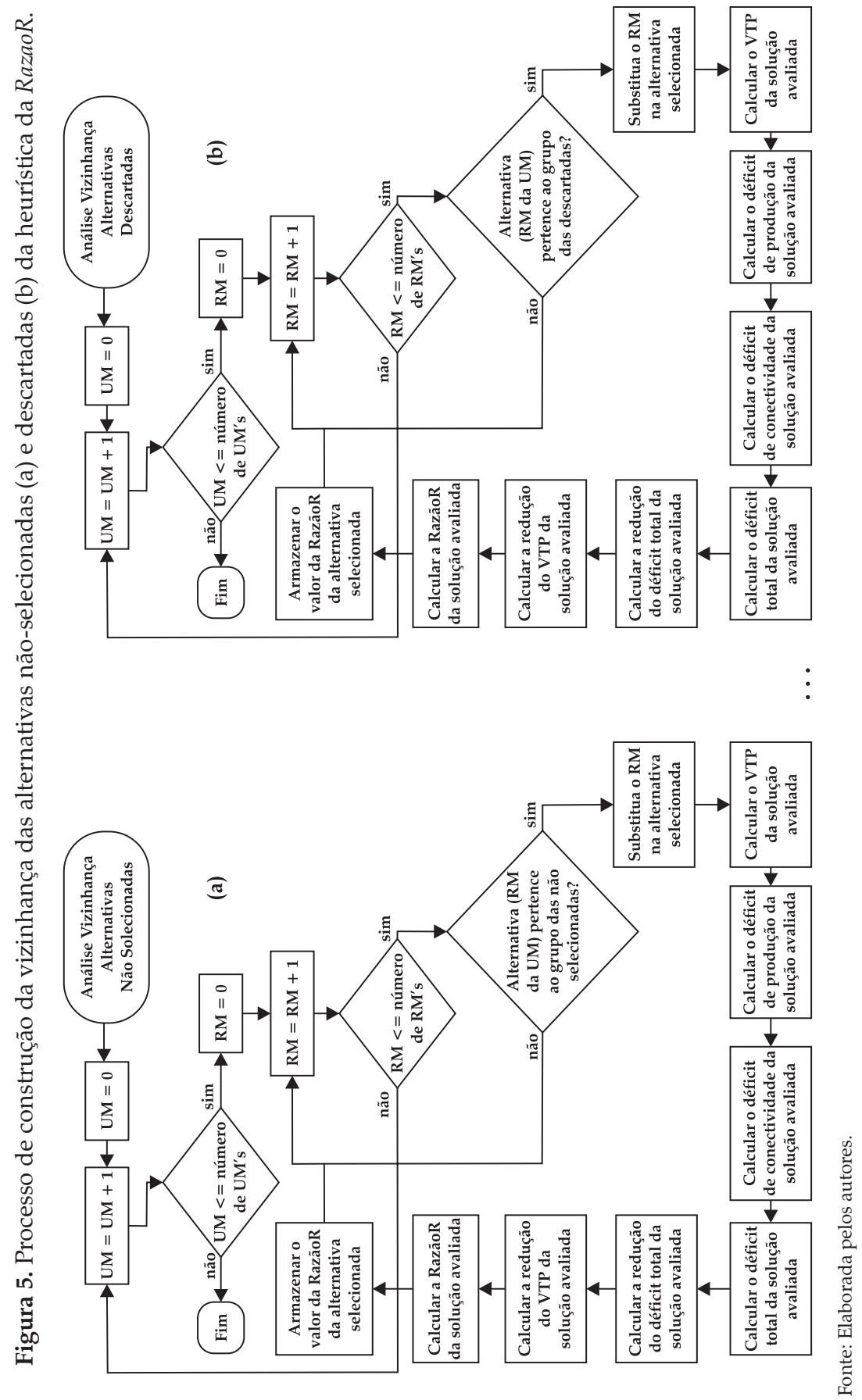


O processo de construção da vizinhança com as alternativas não-selecionadas é realizado ao se substituir, para cada unidade de manejo por vez, o regime de manejo atualmente na base por um regime de manejo pertencente à unidade de manejo avaliada, que esteja no conjunto das alternativas nãoselecionadas. Além disso, calcula-se a diferença do Valor Total do Projeto (VTP), que constitui a função objetivo do problema, e a diferença no déficit total, obtido a partir da soma ponderada dos déficits anuais de produção e conectividade. Com a diferença do VTP e do déficit total, calcula-se a RazaoR para aquela solução avaliada, representada pela alteração do regime de manejo de uma unidade de manejo em comparação com a solução atualmente mantida na base do problema. O processo é repetido para todos os regimes de manejo nãoselecionados, para cada unidade de manejo presente no problema, conforme pode ser observado na Figura 5a. O processo de construção da vizinhança com as alternativas pertencentes ao conjunto das descartadas se dá de forma semelhante ao método com as alternativas não-selecionadas. A diferença é que os regimes de manejo presentes na base são substituídos pelos regimes de manejo presentes no conjunto das alternativas descartadas, como ilustra a Figura $5 b$.

Após a construção da vizinhança da solução, a heurística escolhe a solução vizinha com a melhor RazaoR, substituindo o regime de manejo selecionado na unidade de manejo. Com uma nova solução na base do problema, enviase a alternativa substituída para o conjunto das alternativas descartadas. Em seguida, a heurística verifica se o déficit total em relação às metas de produção e conectividade atingiu o valor zero. Em caso afirmativo, inicia-se a Fase Verde e, em caso negativo, analisa-se se o valor da melhor RazaoR encontrada é superior ao valor do parâmetro SemMelhora, que determina que não há solução vizinha que melhore o valor da solução atualmente mantida na base. Não havendo como melhorar, a heurística apresenta o valor contido na base e encerra o processo de busca; se o valor da melhor RazaoR for superior ao valor do parâmetro SemMelhora, a heurística continua o processo de busca no espaço de soluções viáveis e inviáveis.

A Fase Verde se constitui em um processo de busca no qual a heurística tenta melhorar a solução, aumentando o valor da função objetivo e mantendo o déficit de total nulo. Conforme ilustrado na Figura 6, o processo de busca na Fase Verde mantém a lógica de avaliação da heurística, mas muda a forma de construção da vizinhança e de avaliação da RazaoR. Para a construção da vizinhança, todas as alternativas que não estão presentes na base da solução atual são utilizadas, não havendo diferença entre as alternativas não-selecionadas e descartadas. A heurística prossegue substituindo os regimes de manejo não associados às unidades de manejo e verificando se, ao realizar a substituição, o déficit total se mantém nulo. Em caso afirmativo, a heurística verifica se a substituição proporcionou acréscimo no VTP; se sim, o valor do acréscimo é inserido na 


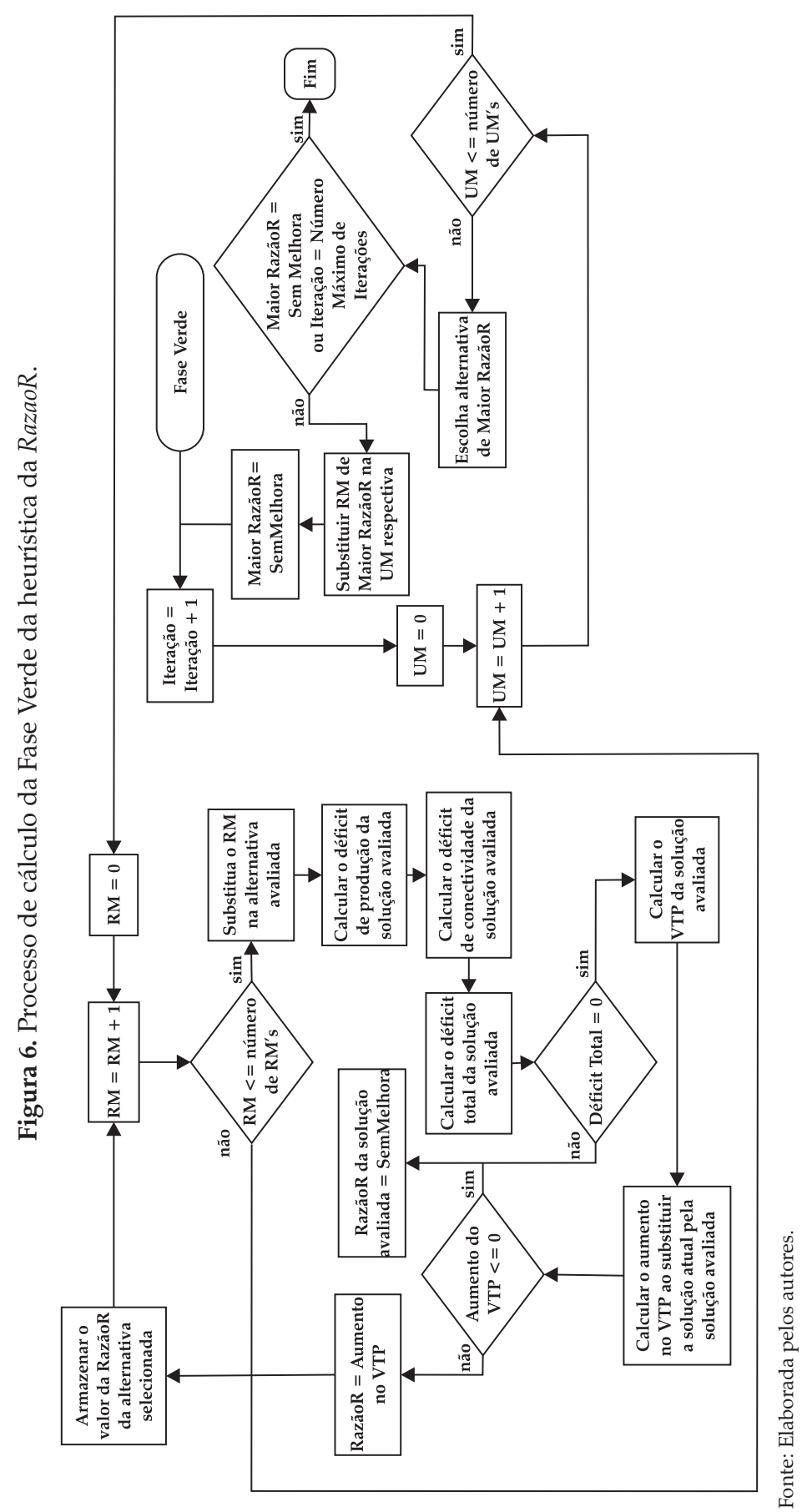

RESR, Piracicaba, SP, vol. 48, no 02, p. 255-282, abr/jun 2010 - Impressa em mês 2010 
RazaoR da solução avaliada. Caso a solução avaliada não mantenha o déficit total nulo ou não proporcione acréscimo no VTP, a RazaoR dessa solução recebe o valor do parâmetro SemMelhora. Após construir toda a vizinhança, a heurística seleciona a alternativa de maior valor da $\operatorname{RazaoR}$ e verifica se esse valor é igual ao parâmetro SemMelhora ou se o número de iterações já atingiu o máximo estipulado pelo usuário. Em caso afirmativo, o processo da Fase Verde se encerra, com a heurística apresentando a melhor solução obtida. Do contrário, a Fase Verde continua até obter uma solução em que o valor da RazaoR seja igual ao parâmetro SemMelhora ou o número máximo de iterações estipulado pelo usuário seja atingido. Após a apresentação da melhor solução obtida pela heurística, o algoritmo é concluído.

\subsection{Materiais utilizados}

A heurística foi programada em um compilador comercial (Visual Basic for Applications - VBA) de simples utilização, integrado a uma planilha de cálculo (MS Excel). O processamento foi realizado em um microcomputador com processador Pentium D 3,4 Ghz, com 4 Gb de memória RAM, e a configuração dos mapas foi feita com o auxílio do software ArcGIS 9.1, possibilitando a visualização dos resultados espaciais obtidos.

\section{Resultados e discussão}

O método proposto para avaliação do déficit de conectividade funcionou corretamente, sendo implantado com sucesso na heurística da RazaoR. A heurística realizou a busca por uma solução viável para o problema reduzindo tanto o déficit de produção quanto o de conectividade. Encontrada a solução, passou-se para a Fase Verde. A solução inicial ${ }^{10}$ apresentou um VTP de R\$ 100.403.284,87, um déficit de produção igual a $272.826,66 \mathrm{~m}^{3}$ de madeira e um déficit de conectividade de 851,68 ha.

Foram efetuadas 140 diferentes estratégias de busca para obtenção de soluções, tendo a heurística encontrado soluções viáveis para todas elas. As estratégias foram elaboradas com a variação dos parâmetros alfa, beta e PesoConec, nos seguintes valores: alfa $=\{10,30,50,70\}$, beta $=\{3,6,9,12,15\}$, e PesoConec $=\{100,600,1.100,3.000,7.000,11.000,15.000\}$.

O problema também foi resolvido com um modelo de programação linear inteira mista, visando obter a solução ótima para comparação com os resultados encontrados pela heurística. A solução ótima apresentou um VTP de R\$ 99.745.798,01, sendo obtida pelo GAMS 22.0 e CPLEX 10.1 após um minuto e 54 segundos de processamento.

10 Melhor solução inteira possível sem se importar com as restrições. 
Para resolução de cada uma das 140 estratégias de busca definidas no trabalho, a macro levou, em média, dez minutos e 25 segundos, totalizando 24 horas, 17 minutos e 25 segundos. A melhor solução obtida foi de $\mathrm{R} \$ 99.576 .615,20$ e a pior, de $\mathrm{R} \$ 99.461 .451,74$, representando 99,83\% e $99,71 \%$ da solução ótima, respectivamente.

A Figura 7 apresenta um histograma contendo a distribuição do número de soluções obtidas pela heurística, classificadas de acordo com faixas prédeterminas de VTP. Verifica-se que $78 \%$ das estratégias executadas retornaram soluções com VTP's variando entre 99,78\% e 99,82\% do valor obtido pela solução ótima, sendo que $62 \%$ dessas estratégias oscilaram de $99,78 \%$ a $99,8 \%$ do valor obtido pela solução ótima.

Figura 7. Histograma do Valor Total do Projeto das estratégias executadas.

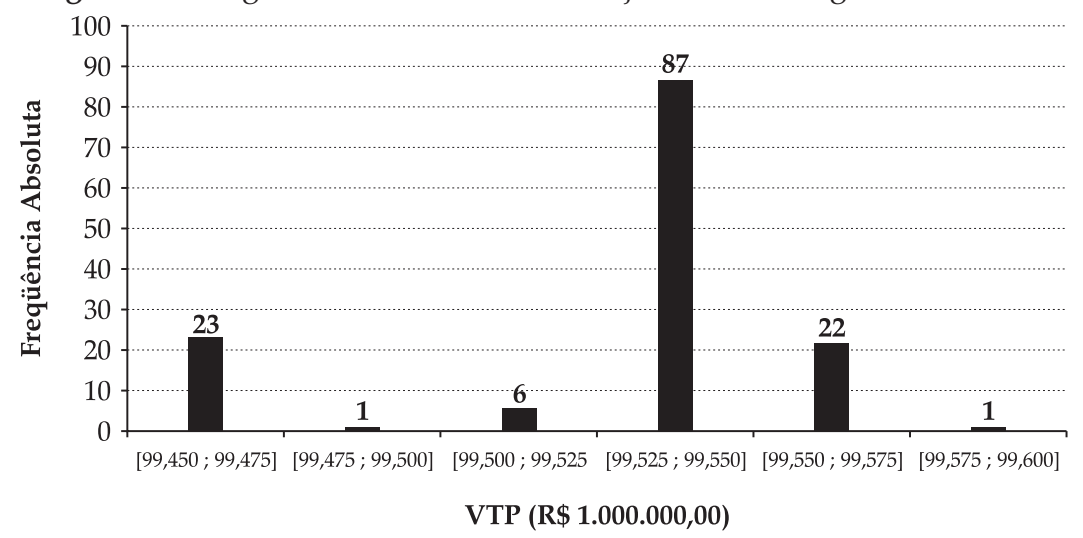

Fonte: Elaborada pelos autores.

De maneira geral, ao observar a Figura 7, percebe-se que a heurística não conseguiu, em nenhuma das estratégias propostas, encontrar a solução ótima para o problema. Entretanto, apresentou ótimas soluções viáveis em todas as estratégias de busca executadas. Prova disso é que a maior diferença no VTP em relação à solução ótima foi de apenas 0,29\%. Dessa forma, o algoritmo proposto mostrou-se bastante eficaz na obtenção de boas soluções viáveis para o problema em questão.

As Figuras 8, 9 e 10 apontam o comportamento da solução obtida em relação às diferentes estratégias elaboradas pela alteração dos valores dos parâmetros PesoConec, Alfa e Beta, respectivamente. 
274 - A Incorporação de Corredores de Conectividade no Manejo de Florestas Industriais Utilizando a Heurística da RazãoR

Figura 8. Comportamento do VTP das soluções em relação ao parâmetro PesoConec.

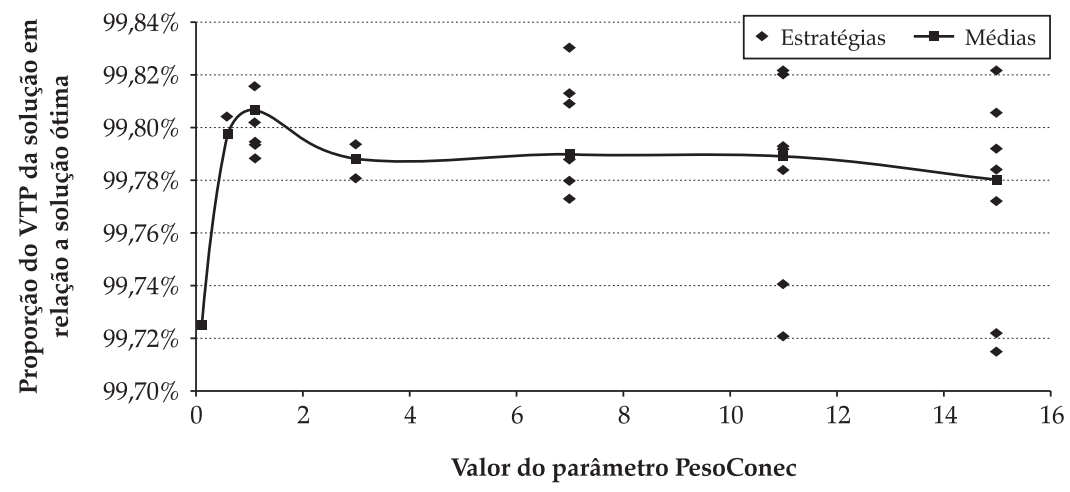

Fonte: Elaborada pelos autores.

Figura 9. Comportamento do VTP das soluções em relação ao parâmetro Alfa.

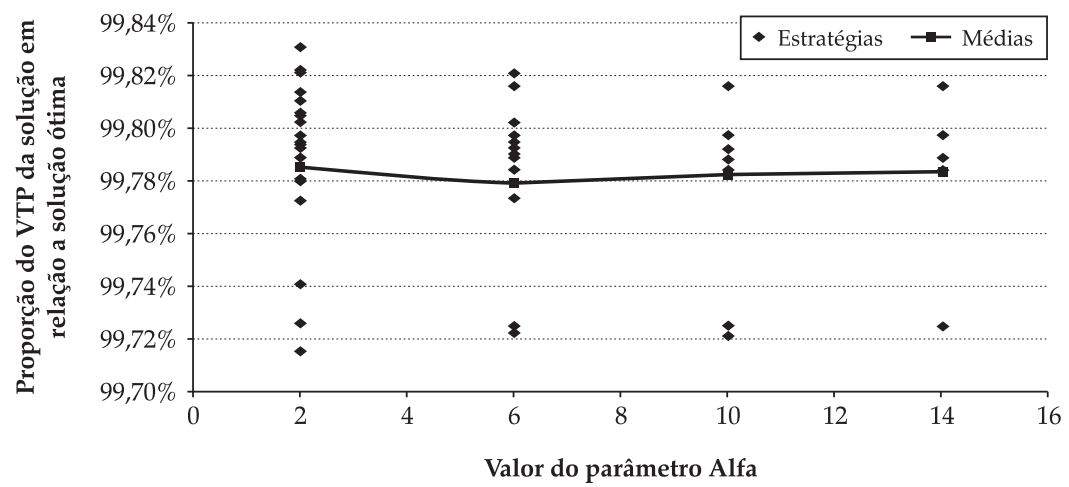

Fonte: Elaborada pelos autores.

Figura 10. Comportamento do VTP das soluções em relação ao parâmetro Beta.

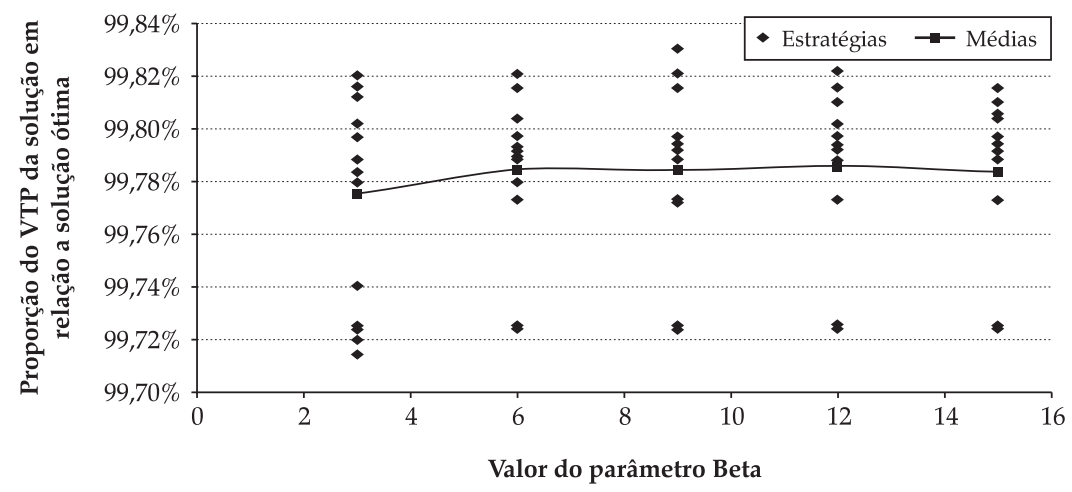

Fonte: Elaborada pelos autores. 
A heurística da RazaoR encontrou melhores soluções para valores do parâmetro PesoConec acima de 600, com a variabilidade dos resultados obtidos aumentando para maiores valores do parâmetro, especialmente a partir de 6.000. Para o problema em questão, valores do PesoConec entre 600 e 3.000 parecem apresentar menor variabilidade do VTP.

A média do VTP registrou pouca oscilação comparada às variações dos parâmetros alfa e beta, conforme pode ser observado nas Figuras 9 e 10, nesta ordem. Entretanto, a maioria das estratégias que apresentaram VTP abaixo de 99,74\% foi obtida com valores de PesoConec igual a 100, sendo este o parâmetro que mais afetou o resultado do VTP encontrado nas diferentes estratégias. Resultaram em soluções de baixo VTP estratégias cujo PesoConec foi igual a 100, havendo uma maior variabilidade das soluções obtidas naquelas em que o valor do parâmetro foi igual ou superior a 7.000. As estratégias cujo valor do PesoConec variou entre 600 e 3.000 refletiram em soluções de boa qualidade e baixa variabilidade, especialmente aquelas com PesoConec igual a 1.100 e valores de alfa iguais ou superiores a 50. Esse resultado confirma a proposta de que é necessário equilibrar os valores dos déficits das diferentes restrições a serem consideradas no problema, de modo a melhorar a eficácia do algoritmo de busca da heurística.

Figura 11. Histograma do número de iterações necessário para execução das estratégias.

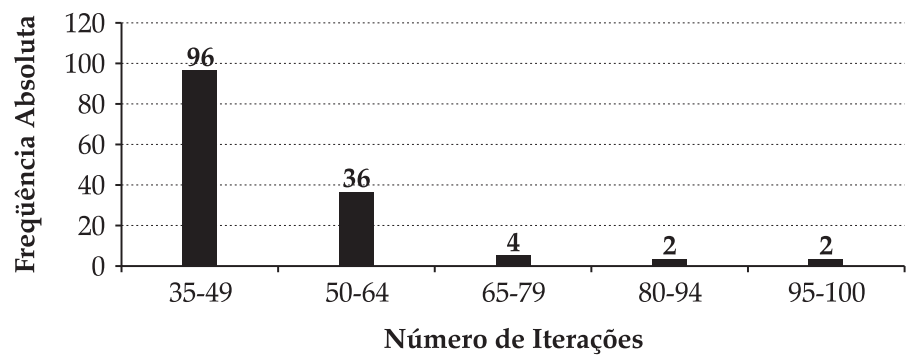

Fonte: Elaborada pelos autores.

Com relação à quantidade de iterações realizadas pela heurística para obter as soluções de cada estratégia, os números em geral foram bastante reduzidos: de 38 a 101, com média de 13 segundos para cada iteração ser executada. Das estratégias, $69 \%$ encontraram boa solução viável com menos de 50 iterações; 94\% das soluções foram obtidas com até 64 iterações, sendo que a melhor solução foi encontrada após 55 iterações da heurística (Figura 11), a partir de estratégia efetuada com Alfa igual a 10, Beta igual a 9 e PesoConec igual a 7.000.

Visando à apresentação da formação dos corredores de conectividade pelas soluções propostas pela heurística, os mapas dos dois primeiros períodos de planejamento foram elaborados para a melhor solução obtida (Figura 12). Nesses mapas, é possível observar que a solução proposta pela heurística obteve sucesso 
na formação dos corredores de conectividade. A imposição de uma produção mínima de $180.000 \mathrm{~m}^{3} /$ ano e de uma produção máxima de 250.000 m³/ano também foi atendida para os 18 anos do horizonte de planejamento (Figura 13).

Figura 12. Mapas dos dois primeiros períodos do plano de manejo resultante da melhor solução encontrada pela heurística da RazaoR.
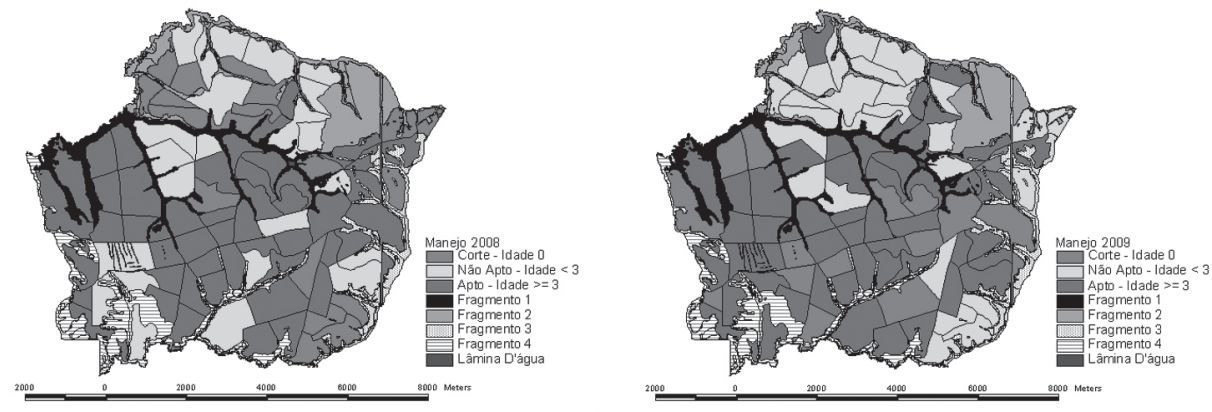

Fonte: Elaborada pelos autores.

Figura 13. Fluxo anual de produção de madeira, obtido pela estratégia com maior VTP encontrada pela heurística da RazaoR.

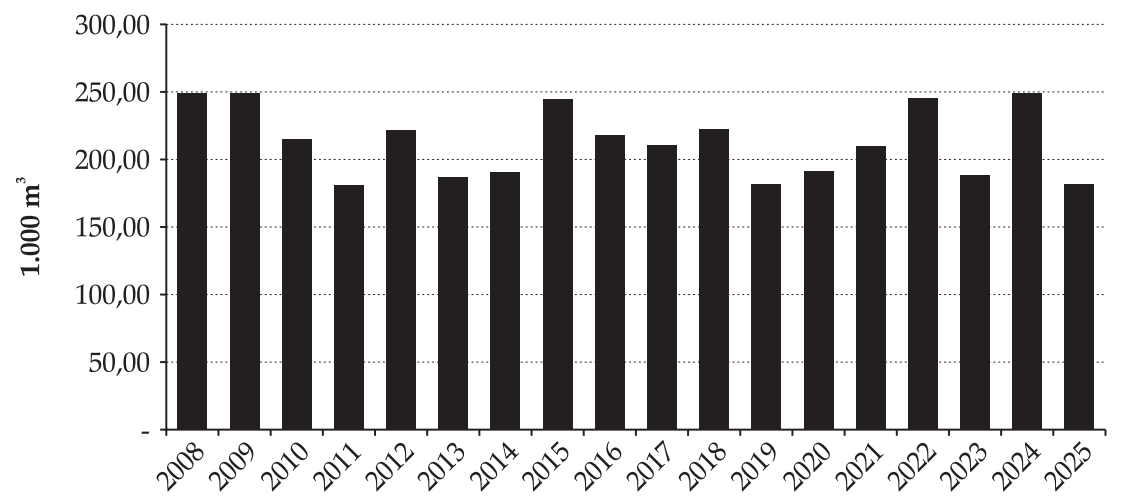

Período do Horizonte de Planejamento

Fonte: Elaborada pelos autores.

De forma geral, a heurística da RazaoR mostrou-se uma estratégia bastante eficaz para resolução de problemas de otimização do planejamento florestal com restrições de conectividade, uma vez que todas as soluções encontradas apresentaram valores superiores a 99,7\% do valor da função objetivo encontrada na solução ótima. Foi também eficiente no número de iterações necessárias para encontrar boas soluções viáveis para o estudo de caso proposto. A heurística 
deixou a desejar, na forma como foi implantada, apenas em termos de tempo de execução, apresentando um esforço computacional superior ao executado pelo modelo matemático de programação linear inteira mista.

O tempo reduzido com que o modelo matemático encontra a solução ótima e o baixo número de iterações necessárias para a heurística obter boas soluções viáveis se devem ao fato de o modelo atender às restrições impostas no problema com relativa facilidade, decorrente da combinação de duas principais características.

A primeira é o elevado número de regimes de manejo com os quais o modelo matemático e a heurística podem trabalhar para encontrar soluções. A distribuição e localização das unidades de manejo e dos fragmentos a serem conectados, aliadas ao grafo proposto e à facilidade para que essas unidades de manejo apresentemse aptas a fazer parte do corredor constituem a segunda característica que facilita o atendimento das restrições do problema, particularmente para a formação dos corredores. Os fragmentos de vegetação nativa da fazenda considerada no estudo de caso permeiam a matriz do plantio de eucalipto. Como se considerou que a elaboração de um caminho entre dois fragmentos era suficiente para o estabelecimento de um corredor de conectividade, não foi difícil para a heurística encontrar soluções viáveis e para o modelo matemático obter a solução ótima.

Embora a heurística tenha mostrado desempenho inferior em termos de tempo de execução, frente ao modelo matemático, tal comparação não deve ser considerada definitiva. Isso porque a implantação do algoritmo da heurística não foi realizada com o enfoque em velocidade de execução, restando boas possibilidades para a melhoria de sua performance.

Além disso, apesar de a fazenda considerada no estudo de caso possuir tamanho e número de unidades de manejo suficientes para se avaliar a aplicabilidade das técnicas propostas para a implantação dos corredores de conectividade em problemas de escala real, sua área ainda é pequena quando comparada à área total média que as empresas de grande porte necessitam trabalhar no planejamento estratégico do manejo de suas florestas. Para esses casos, modelos matemáticos de programação linear inteira mista podem não ser capazes de gerar soluções viáveis em um tempo computacionalmente aceitável. É, portanto, papel da ciência elaborar e testar métodos alternativos, como a heurística da RazaoR, que permitam a gestores florestais resolverem problemas de otimização.

\section{Conclusões}

O algoritmo recursivo utilizado na programação dinâmica mostrou-se um bom método de avaliação do déficit de conectividade para as soluções propostas. Adequou-se perfeitamente à estratégia de busca utilizada pela heurística da RazaoR após a inserção do parâmetro de ponderação entre o déficit de conectividade e o déficit de produção. 
A heurística apresentou-se eficaz na obtenção de boas soluções viáveis com um baixo número de iterações. O desempenho em termos de tempo de execução do algoritmo pode ser melhorado com a alteração da linguagem e, principalmente, do código de programação, além da criação de um programa executável. Isso reduziria o tempo para a obtenção de soluções viáveis, possibilitando utilizar a heurística para resolução de problemas de maior porte.

Para estudos futuros, recomenda-se o desenvolvimento de outros algoritmos para calcular o déficit de conectividade das soluções avaliadas, com destaque àqueles que permitam o uso de arcos bidirecionados no grafo representando os possíveis caminhos para a formação dos corredores. Outra linha a ser seguida consiste na elaboração de formas mais eficientes para avaliar a vizinhança da heurística, focando uma vizinhança parcial com as melhores alternativas a serem consideradas, de modo a reduzir o tempo de execução de cada iteração. Como consequência, o tempo necessário para o algoritmo encontrar soluções viáveis também diminuiria.

A heurística da RazaoR proposta neste trabalho ainda pode ser utilizada por gestores florestais que não disponham dos softwares necessários para resolução de problemas de programação linear inteira mista em larga escala, principalmente considerando a formação de corredores de conectividade.

\section{Referências Bibliográficas}

AMERICAN FOREST AND PAPER ASSOCIATION. Sustainable Forest Initiative Standard - Principles and Objectives. Washington: AF\&PA, 2000, 7 p. Disponível em: < http://64.29.218.140/Content/NavigationMenu/Environment_and_ Recycling/SFI/Publications1/2000_standard.pdf >. Acesso em: 13 mar 2006.

ARRUDA, M.B. Corredores ecológicos do brasil-gestão integrada de ecossistemas. In: ARRUDA, M.B.; NOGUEIRA, L.F.S. Corredores ecológicos: uma abordagem integradora de ecossistemas no Brasil. Brasília: IBAMA, 2004. p. 11-46.

AUGUSTI, J.C.; FERRAZ, F.F.de; Ferraz, S.F.de; Silva, J.F.da; Camargo, S.L. Cultivo integrado com florestas naturais de baixo impacto. Visão Agrícola, Piracicaba, v.4, p. 62-65, dez 2005.

BEIER, P; NOSS, R.F. Do habitat corridors provide connectivity? Conservation Biology, Oxford, v. 12, n. 6, p. 1241-1252, Dec 1998.

BORGES, J.G.; HOGANSON, H.M. Assessing the impact of management unit design and adjacency constraints on forestwide spatial conditions and timber revenues. Canadian Journal of Forest Research, Ottawa, v. 29, n. 11, p. 1764-1774, 1999.

CARO, F.; CONSTANTINO, M.; MARTINS, I.; WEINTRAUB, A. A 2-Opt Tabu Search Procedure for the Multiperiod Forest Harvesting Problem with 
Adjacency, Greenup, Old Growth, and Even Flow Constraints. Forest Science, Bethesda, v. 49, n. 5, p. 738-750, Oct 2003.

CERDEIRA, J.O. GASTON, K.J.; PINTO, L.S. Connectivity in priority area selection for conservation. Enviromental Modelling and Assessment, Amsterdam, v.10, n. 3, 2005. Diponível em: <http://www.springerlink.com.w10077.dotlib. com.br/content/u8654v14736176qr/?p=63fe7f7fc01248718f567fdf10eefbf2\& pi $=2>$. Acesso em: 15 jun 2006.

CLUTTER, J.L.; FORTSON, J.C.; PIENAAR, L.V.; BRISTER, G.H.; BAILEY, R.L. Timber management: a quantitative approach. Florida: Krieger Publishing Company, 1992. 333p.

FALCÃO, A.O.; BORGES, J. Combining Random and Systematic Search Heuristic Procedures for Solving Spatially Constrained Forest Management Scheduling Models. Forest Science, Bethesda, v. 48, n. 3, p. 608-620, Aug 2002.

FONSECA, G.A.B.; ALGER, K.; PINTO, L.P.; ARAÚJO, M.; CAVALCANTI, R. Corredores de Biodiversidade: O Corredor Central da Mata Atlântica. In: ARRUDA, M.B.; NOGUEIRA, L.F.S. Corredores ecológicos: uma abordagem integradora de ecossistemas no Brasil. Brasília: IBAMA, 2004. p. 47-66.

FULLER, T.; SARKAR, S. LQGraph: A software package for optimizing connectivity in conservation planning. Environmental Modelling $\mathcal{E}$ Software, Amsterdam, v. 21, n. 5, p. 750-755, May 2006.

GARCIA, J.N.; BORTOLLETO JÚNIOR, G. Otimização da matéria-prima "árvore" deve ser prioridade. Visão Agrícola, Piracicaba, v. 4, p. 92-96, jul - dez 2005.

GOYCOOLEA, M.; MURRAY, A.T.; BARAHONA, F.; EPSTEIN, R.; WEINTRAUB, A. Harvest Scheduling Subject to Maximum Area Restrictions: Exploring Exact Approaches. Operations Research, Cambridge, v. 53, n. 3, p. 490-500, May-Jun 2005.

HESS, G. R. Conservation corridors and contagious disease: a cautionary note. Conservation Biology, Oxford, v. 8, n. 1, p. 256-262, May 1994.

HILLIER, F.S; LIEBERMAN, G.J. Introduction to operations research. $8^{\text {th }}$ ed. New York: McGraw-Hill, 2005. 1061 p.

HOGANSON, H.M.; BORGES, J.G. Using Dynamic Programming and Overlapping Subproblems to Address Adjacency in Large Harvest Scheduling Problems. Forest Science, Bethesda, v. 44, n. 4, p. 526-538, Nov 1998.

HORSKINS, K.; MATHER, P.B.; WILSON, J.C. Corridors and connectivity: when use and function do not equate. Landscape Ecology, Dordrecht, v. 21, n. 5, Jul 2006. Disponível em: < http://www.springerlink.com.w10077.dotlib.com.br/ content/84qq533865702442/fulltext.pdf>. Acesso em: 20 nov 2006. 
A Incorporação de Corredores de Conectividade no Manejo de Florestas Industriais Utilizando a Heurística da RazãoR

INFORMATIVO CEPEA - SETOR FLORESTAL. Piracicaba, n. 59, nov 2006. 4 p. Disponível em : <http://www.cepea.esalq.usp.br/pdf/inform59.pdf > . Acesso em: 20 dez 2006.

LOCKWOOD, C.; MOORE, T. Harvest scheduling with spatial constraints: a simulated annealing approach. Canadian Journal of Forest Research, Ottawa, v. 23, n. 3, p. 468-478, Mar 1993.

LOEHLE, C. Optimizing wildlife habitat mitigation with a habitat defragmentation algorithm. Forest Ecology and Management, Amsterdam, v.120, p. 245-251, 1999.

MARTELL, D.L.; GUNN, E.A.; WEINTRAUB, A. Forest management challenges for operational researchers. European Journal of Operational Research, Amsterdam, v. 104, n. 1, p. 1-17, Jan 1998.

MAURER, S.B.; RALSTON, A. Discrete algorithmic mathematics. $3^{\text {rd }}$ ed. Wellesley: A K Peters, 2004. 772 p.

McDILL, M.E.; BRAZE, J. Comparing Adjacency Constraint Formulations for Randomly Generated Forest Planning Problems with Four Age-Class Distributions. Forest Science, Bethesda, v. 46, n. 3, p. 423-436, Aug 2000.

McDILL, M.E.; BRAZE, J. Using the Branch and Bound Algorithm to Solve Forest Planning Problems with Adjacency Constraints. Forest Science, Bethesda, v. 47, n. 3, p. 403-418, Aug 2001.

McDILL, M.E.; REBAIN, S.A.; BRAZE, J. Harvest Scheduling with Area-Based Adjacency Constraints. Forest Science, Bethesda, v. 48, n. 4, p. 631-642, Nov 2002.

MEYER, W. B.; TURNER II, B. L. Changes in land use and land cover: a global perspective. New York: Cambridge University Press, 1994. 537 p.

MURRAY, A.T. Spatial restrictions in harvest scheduling. Forest Science, Bethesda, v. 45, n. 1, p. 45-52, Feb 1999.

NOBRE, S.R. A heurística da Razão-R aplicada a problemas de gestão florestal. 1999.129 p. Dissertação (Mestrado em Administração Rural) - Universidade Federal de Lavras, 1999.

NOBRE, S.R.; RODRIGUEZ, L.C.E. Analysis and evaluation of the R-Ratio heuristic for forest scheduling. BEVERS, MICHAEL; BARRETT, TARA M.(Comp.).: In: SYMPOSIUM SYSTEMS ANALYSIS IN FOREST RESOURCES; 2003. Stevenson, Oct 7-9, Stevenson, WA. Portland, OR: USDA Forest Service, 2005. p. 287-295. USDA Forest Service - General Techical Report PNW-GTR-000

ÖHMAN, K. Creating continuous areas of old forest in long-term forest planning. Canadian Journal of Forest Research, Ottawa, v. 30, n. 11, p. 1817-1823, Nov 2000. 
ÖHMAN, K.; ERIKSSON, L.O. Allowing for spatial consideration in long-term forest planning by linking linear programming with simulated annealing. Forest Ecology and Management, Amsterdam, v. 161, n. 1/3, p. 221-230, May 2002.

ÖHMAN, K.; ERIKSSON, L.O. The core area concept in forming contiguous areas for long-term forest planning. Canadian Journal of Forest Research, Ottawa, v. 28, n. 7, p. 1032-1039, Jul 1998.

ÖNAL, H.; BRIERS, R.A. Designing a conservation reserve network with minimal fragmentation: A linear integer programming approach. Environmental Modeling and Assessment, Amsterdan, v. 10, n. 3, Sep 2005. Disponível em: <http:// www.springerlink.com/content/k1q2527g368u043g/fulltext.pdf > . Acesso em: 16 de sep 2006.

PRIMACK, R.B.; RODRIGUES, E. Biologia da conservação. Londrina: E. Rodrigues, 2001. 328p.

REBAIN, S.; McDILL, M.E. A mixed-integer formulation of the minimum patch size problem. Forest Science, Bethesda, v. 49, n. 4, p. 608-618, Aug 2003.

RODRIGUEZ, L.C.E. Use of heuristic methods in the analysis of large-scale forest management integer programming models. 1994. 93p. Tesis (Doctor of Philosophy in Forest Management and Biometrics) - University of Georgia, Athens. 1994.

SESSIONS, J. Solving for habitat connections as a steiner network problem. Forest Science, Bethesda, v. 38, n. 1, p.203-207, Feb 1992.

WEINTRAUB, A.; BAHARONA, F.; EPSTEIN, R. A Column Generation Algorithm for Solving General Forest Planning Problems with Adjacency Constraints. Forest Science, Bethesda, v. 40, n. 1, p. 142-161, Feb 1994.

WILLIAMS, J.C. Delineating protected wildlife corridors with multi-objective programming. Environmental Modeling and Assessment, Amsterdam, v. 3, n. 1/2, p. 77-86, Mar 1998.

WINSTON, W.L. Operations Research: Applications and Algorithms. $4^{\text {th }}$ ed. Canada: THOMSON Brooks/Cole, 2004. 1434p. 
